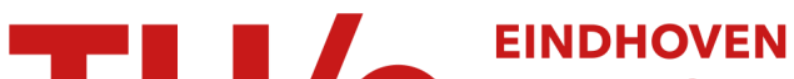 UNIVERSITY OF TECHNOLOGY
}

\section{An entropy theorem for computing the capacity of weakly (d, k)-constrained sequences}

\section{Citation for published version (APA):}

Janssen, A. J. E. M., \& Schouhamer Immink, K. A. (2000). An entropy theorem for computing the capacity of weakly (d, k)-constrained sequences. IEEE Transactions on Information Theory, 46(3), 1034-1038. https://doi.org/10.1109/18.841180

DOI:

10.1109/18.841180

Document status and date:

Published: 01/01/2000

\section{Document Version:}

Publisher's PDF, also known as Version of Record (includes final page, issue and volume numbers)

\section{Please check the document version of this publication:}

- A submitted manuscript is the version of the article upon submission and before peer-review. There can be important differences between the submitted version and the official published version of record. People interested in the research are advised to contact the author for the final version of the publication, or visit the $\mathrm{DOI}$ to the publisher's website.

- The final author version and the galley proof are versions of the publication after peer review.

- The final published version features the final layout of the paper including the volume, issue and page numbers.

Link to publication

\section{General rights}

Copyright and moral rights for the publications made accessible in the public portal are retained by the authors and/or other copyright owners and it is a condition of accessing publications that users recognise and abide by the legal requirements associated with these rights.

- Users may download and print one copy of any publication from the public portal for the purpose of private study or research.

- You may not further distribute the material or use it for any profit-making activity or commercial gain

- You may freely distribute the URL identifying the publication in the public portal.

If the publication is distributed under the terms of Article $25 \mathrm{fa}$ of the Dutch Copyright Act, indicated by the "Taverne" license above, please follow below link for the End User Agreement:

www.tue.nl/taverne

Take down policy

If you believe that this document breaches copyright please contact us at:

openaccess@tue.nl

providing details and we will investigate your claim. 


\section{An Entropy Theorem for Computing the Capacity of Weakly $(d, k)$-Constrained Sequences}

\author{
Augustus J. E. M. Janssen, Senior Member, IEEE, and \\ Kees A. Schouhamer Immink, Fellow, IEEE
}

\begin{abstract}
In this correspondence we find an analytic expression for the maximum of the normalized entropy $-\sum_{i \in T} p_{i} \ln p_{i} / \sum_{i \in T} i p_{i}$ where the set $T$ is the disjoint union of sets $S_{n}$ of positive integers that are assigned probabilities $P_{n}, \sum_{n} P_{n}=1$. This result is applied to the computation of the capacity of weakly $(d, k)$-constrained sequences that are allowed to violate the $(d, k)$-constraint with small probability.
\end{abstract}

Index Terms-Capacity, constrained code, $(d, k)$ sequence, entropy, magnetic recording, $R L L$ sequence, runlength-limited.

\section{INTRODUCTION AND ANNOUNCEMENT OF RESULTS}

Let $T$ be a set of positive integers, and assume that $T$ is the disjoint union of a (finite or infinite) number of nonempty sets $S_{n}, n \in M$. Also assume that there are given numbers $P_{n} \geq 0, n \in M$, with $\sum_{n} P_{n}=1$. We show the following result

Theorem: The maximum of

$$
H:=\frac{-\sum_{i \in T} p_{i} \ln p_{i}}{\sum_{i \in T} i p_{i}}
$$

under the constraints that $p_{i} \geq 0$ and

$$
\sum_{i \in S_{n}} p_{i}=P_{n}, \quad n \in M
$$

equals $z_{0}$, where $z_{0}>0$ is the unique solution $z$ of the equation

$$
-\sum_{n \in M} P_{n} \ln Q_{n}(z)=-\sum_{n \in M} P_{n} \ln P_{n}
$$

with $Q_{n}(z)$ given for $z>0$

$$
Q_{n}(z):=\sum_{i \in S_{n}} e^{-i z}, \quad n \in M .
$$

Moreover, the optimal $p_{i}$ are given by

$$
p_{i}=\frac{P_{n}}{Q_{n}\left(z_{0}\right)} e^{-i z_{0}}, \quad i \in S_{n}, n \in M
$$

and for these $p_{i}$ we have that

$$
\sum_{i \in T} i p_{i}=\frac{d}{d z}\left[-\sum_{n \in M} P_{n} \ln Q_{n}(z)\right]\left(z_{0}\right) .
$$

As an application of this result we consider weakly constrained $(d, k)$ sequences [1]. A binary $(d, k)$-constrained sequence has by definition at least $d$ and at most $k$ "zeros" between consecutive "ones." Such sequences are applied in mass storage devices such as the compact disc (CD) and the DVD. Weakly constrained codes do not strictly work to the rules, as they produce sequences that violate the specified constraints with a given (small) probability, see Section III for an explicit description. It is argued that if the channel is not free of errors, it is pointless to feed the channel with perfectly constrained

Manuscript received May 20, 1999; revised November 19, 1999. The material in this correspondence will be presented at the IEEE International Symposium on Information Theory, Sorrento, Italy, June 25-30, 2000.

A. J. E. M. Janssen is with the Philips Research Laboratories, WY 81, 5656 AA Eindhoven, The Netherlands (e-mail: A.J.E.M.Janssen@philips.com).

K. A. S. Immink is with the Institute for Experimental Mathematics, 45326 Essen, Germany (e-mail: immink@exp-math.uni-essen.de).

Communicated by E. Soljanin, Associate Editor for Coding Techniques.

Publisher Item Identifier S 0018-9448(00)02898-4. sequences. Clearly, the extra freedom will result in an increase of the channel capacity. A $(d, k)$-constrained sequence can be thought to be composed of "phrases" $10^{i}, d \leq i \leq k$, where $0^{i}$ means a series of $i$ "zeros." In order to compute the channel capacity, i.e., the maximum $z_{0} / \ln 2$ of the entropy $H / \ln 2$, we define

$$
\begin{aligned}
T & =\{1, \cdots, d\} \cup\{d+1, \cdots, k+1\} \cup\{k+2, k+3, \cdots\} \\
& =: S_{1} \cup S_{2} \cup S_{3}
\end{aligned}
$$

where $d=0,1, \cdots$, and $k=d+1, d+2, \cdots$ are given, and we compute the capacity for the case that the probabilities $P_{1}, P_{3}$ assigned to the sets $S_{1}, S_{3}$ are both small. Clearly, the quantities $P_{1}$ and $P_{3}$ denote the probabilities that phrases are transmitted that are either too short or too long, respectively. We find that the familiar capacities of $(d, k)$-constrained sequences [2] are approached from above as $P_{1}$, $P_{3} \rightarrow 0$ with an error $A\left(P_{1} \ln P_{1}+P_{3} \ln P_{3}\right)$, where we can evaluate the $A$ explicitly. We obtain a similar result for the case that $T$ is as in (6) with $S_{1}, S_{3}$ merged into a single set $S_{1} \cup S_{3}$.

\section{PROOF OF THE THEOREM}

We present the proof of the theorem for the case that the set $T$, and consequently the sets $M$ and $S_{n}, n \in M$, are finite. The case that some of these sets may be infinite gives no particular problems, but complicates the presentation given below somewhat. At the end of this section, we shall indicate some modifications that are needed to have the argument work for this more general case as well.

The plan of the proof is as follows. We fix $x>0$ in a range $\left[x_{-}, x_{+}\right]$ to be specified below, and we maximize, using Lagrange's theorem, the quantity

$$
\frac{-1}{x} \sum_{i \in T} p_{i} \ln p_{i}
$$

over $p_{i} \geq 0$ under the constraints that

$$
\sum_{i \in T} i p_{i}=x, \quad \sum_{i \in S_{n}} p_{i}=P_{n}, \quad n \in M .
$$

The maximum value of (7) thus obtained is maximized over $x \in\left[x_{-}, x_{+}\right]$and this yields the maximum $H$ in (1) under the constraints on the $p_{i}$ in the theorem.

The range of $x$ to be considered in (7) and (8) is equal to $\left[x_{-}, x_{+}\right]$, where

$$
x_{-}=\sum_{n \in M} P_{n} \min _{i \in S_{n}} i \quad x_{+}=\sum_{n \in M} \frac{P_{n}}{\left|S_{n}\right|} \sum_{i \in S_{n}} i .
$$

To see this, we observe that for any choice of $p_{i}, i \in T$, satisfying $\sum_{i \in S} p_{i}=P_{n}$, we can increase the value of $H$ in (1) by ordering the $p_{i}$ 's per set $S_{n}$ decreasingly. Indeed, this does not change the values of $-\sum_{i \in T} p_{i} \ln p_{i}$ and $\sum_{i \in S_{n}} p_{i}, n \in M$, while it decreases the value of $\sum_{i \in T} i p_{i}$. Now $x_{-}$corresponds to the case that all mass $P_{n}$ of $S_{n}$ is assigned to the minimal element of $S_{n}, n \in M$, while $x_{+}$corresponds to the case that all elements of $S_{n}$ are assigned equal masses $\left|S_{n}\right|^{-1} P_{n}$.

To minimize (7) under the constraint (8), we observe that (7) is a continuous, strictly concave function of the $p_{i}$ 's restricted to the convex set described by (8) and $p_{i} \geq 0, i \in T$. Hence the maximum of (7) under the given constraints exists and is unique. By applying Lagrange's multiplier rule, we easily find that the maximizing $p_{i}$ are of the form

$$
p_{i}=e^{-\left(\lambda i+\mu_{n}\right) x-1}, \quad i \in S_{n}, n \in M
$$

with the Lagrange multipliers $\lambda$ and $\mu_{n}, n \in M$, corresponding to the first constraint and the second constraints in (8), respectively, such that the $p_{i}$ 's in (10) satisfy (8). From what has been said above we have $\lambda \geq 0$. 
It is easy to show that the constraints (8) imply that

$$
\mu_{n} x+1=\ln \left[Q_{n}(\lambda x) / P_{n}\right], \quad n \in M
$$

with $Q_{n}$ given in (3), and that

$$
x=\sum_{n} P_{n} \frac{R_{n}(\lambda x)}{Q_{n}(\lambda x)}
$$

with $R_{n}$ given for $z \geq 0$ by

$$
R_{n}(z):=\sum_{i \in S_{n_{2}}} i e^{-i z}=-Q_{n}^{\prime}(z), \quad n \in M .
$$

We shall now show that for any $x \in\left(x_{-}, x_{+}\right]$there is a unique solution $\lambda \in[0, \infty)$ of (12). Indeed, we have for $x>0$ fixed that

$$
\frac{d}{d \lambda}\left[\frac{R_{n}(\lambda x)}{Q_{n}(\lambda x)}\right]=x \frac{R_{n}^{\prime}(\lambda x) Q_{n}(\lambda x)-R_{n}(\lambda x) Q_{n}^{\prime}(\lambda x)}{Q_{n}^{2}(\lambda x)}
$$

and for $n \in M, z \geq 0$

$$
\begin{aligned}
R_{n}^{\prime}(z) Q_{n}(z) & -R_{n}(z) Q_{n}^{\prime}(z) \\
= & -\sum_{i \in S_{n}} i^{2} e^{-i z} \sum_{i \in S_{n}} e^{-i z}+\left(\sum_{i \in S_{n}} i e^{-i z}\right)^{2} \leq 0
\end{aligned}
$$

by the Cauchy-Schwarz inequality with equality if and only if $S_{n}$ is a singleton. Also

$$
\sum_{n} P_{n} \frac{R_{n}(0)}{Q_{n}(0)}=x_{+} \quad \lim _{\lambda \rightarrow \infty} \sum_{n} P_{n} \frac{R_{n}(\lambda x)}{Q_{n}(\lambda x)}=x_{-} .
$$

Hence, except in the trivial case that all $S_{n}$ 's with $P_{n}>0$ are singletons, the right-hand side function in (12) strictly decreases from $x_{+}$at $\lambda=0$ to $x_{-}$at $\lambda=\infty$, as required.

Denoting the unique solution of $\lambda$ of (12) by $\lambda(x)$ for $x \in\left(x_{-}, x_{+}\right]$, we find from (11) and (12) for the maximum value of (7) under the constraints (8) that

$$
H(x)=\lambda(x) x-\frac{1}{x} \sum_{n} P_{n} \ln P_{n}+\frac{1}{x} \sum_{n} P_{n} \ln Q_{n}(\lambda(x) x) .
$$

To maximize $H(x)$ over $x \in\left(x_{-}, x_{+}\right]$we differentiate $H(x)$ with respect to $x$, and we get using (13)

$$
\begin{aligned}
H^{\prime}(x)= & (\lambda(x) x)^{\prime}+\frac{1}{x^{2}} \sum_{n} P_{n} \ln P_{n} \\
& +\frac{-1}{x^{2}} \sum_{n} P_{n} \ln Q_{n}(\lambda(x) x) \\
& -\frac{1}{x}(\lambda(x) x)^{\prime} \sum_{n} P_{n} \frac{R_{n}(\lambda(x) x)}{Q_{n}(\lambda(x) x)} .
\end{aligned}
$$

By (12) and the definition of $\lambda(x)$ it thus follows that

$$
H^{\prime}(x)=\frac{1}{x^{2}}\left(\sum_{n} P_{n} \ln P_{n}-\sum_{n} P_{n} \ln Q_{n}(\lambda(x) x)\right) .
$$

We shall next show that there is a unique $x_{0} \in\left(x_{-}, x_{+}\right)$such that

$$
H^{\prime}\left(x_{0}\right)=0 ; \quad H^{\prime}(x)>0, x<x_{0} ; \quad H^{\prime}(x)<0, x>x_{0} .
$$

We first observe that $\lambda(x) x$ decreases from $\infty$ to 0 as $x$ increases from $x_{-}$to $x_{+}$. Indeed, from (12) and the definition of $\lambda(x)$ we have

$$
\begin{aligned}
1 & =\sum_{n} P_{n} \frac{d}{d x}\left[\frac{R_{n}(\lambda(x) x)}{Q_{n}(\lambda(x) x)}\right] \\
& =(\lambda(x) x)^{\prime} \sum_{n} P_{n}\left(\frac{d}{d z} \frac{R_{n}(z)}{Q_{n}(z)}\right)(z=\lambda(x) x) .
\end{aligned}
$$

As in (14) and (15) we have that $\left(R_{n}(z) / Q(z)\right)^{\prime} \leq 0$, with equality signs for all $n$ only in trivial cases, whence $(\lambda(x) x)^{\prime}<0$. Also, it is easy to see that

$$
\lim _{x \uparrow x_{+}} \lambda(x) x=0 \quad \lim _{x \downarrow x_{-}} \lambda(x) x=\infty
$$

as required. Hence, except in trivial cases, we have that $\sum_{n} P_{n} \ln Q_{n}(\lambda(x) x)$ increases from $\infty$ to

$$
\sum_{n} P_{n} \ln \left|S_{n}\right| \geq 0>\sum_{n} P_{n} \ln P_{n}
$$

as $x$ increases from $x_{-}$to $x_{+}$. Therefore, there is a unique $x_{0}$ such that (20) holds.

Evidently, $H(x)$ assumes its maximum at the $x_{0}$ of the previous paragraph, and we have at this $x_{0}$ from (17) that

$$
H\left(x_{0}\right)=\lambda\left(x_{0}\right) x_{0}=: z_{0} .
$$

Thus we see that the maximum of $H(x)$ over $x \in\left(x_{-}, x_{+}\right]$equals $z_{0}$, where $z_{0}$ is the unique solution $z=\lambda(x) x$ of the equation

$$
-\sum_{n} P_{n} \ln Q_{n}(z)=-\sum_{n} P_{n} \ln P_{n}
$$

This proves (2) of the theorem. From (12) and (13) and the definition of $\lambda(x)$ we get the formula (5), and the explicit expression for the $p_{i}$ in (4) follows from (10). This completes the proof of the theorem.

We now briefly comment on the required modifications to have the argument of the proof also work for the case that some of the sets $S_{n}$ are infinite. Now $x_{+}=\infty$, and we must consider $x \in\left(x_{-}, x_{+}\right)$. Also, for $x \in\left(x_{-}, \infty\right)$ fixed, the right-hand side of (12) strictly decreases in $\lambda$ from $\infty$ at $\lambda=0$ to $x_{-}$at $\lambda=\infty$, whence there is a unique solution $\lambda=\lambda(x)$ of (12). Finally, the maximization of $H(x)$ over $x \in\left(x_{-}, \infty\right)$ can be done in a similar way as in the case of finite $T$.

\section{Application to Weakly $(d, k)$-Constrained Sequences}

We shall now apply our theorem to the computation of the capacity of weakly $(d, k)$-constrained sequences, these being allowed to violate the $(d, k)$-constraint with (small) probability. Accordingly, we let $d, k$ be two nonnegative integers, $k>d$ (with $k$ possibly $\infty$ ), and we consider the set $T=\{1,2, \cdots\}$ partitioned as

$$
\begin{aligned}
T & =\{1, \cdots, d\} \cup\{d+1, \cdots, k+1\} \cup\{k+2, k+3, \cdots\} \\
& =S_{1} \cup S_{2} \cup S_{3}
\end{aligned}
$$

where the sets $S_{n}, n=1,2,3$ are assigned probabilities $P_{n} \geq 0$ with $P_{1}+P_{2}+P_{3}=1$. For this kind of application it is customary to consider the normalized entropy

$$
\bar{H}=\frac{-\sum_{i} p_{i} \log _{2} p_{i}}{\sum_{i} i p_{i}}=\frac{1}{\ln 2} H
$$

with $H$ of (1).

We compute for $z>0$

$$
\begin{aligned}
& Q_{1}(z)=\sum_{i=1}^{d} e^{-i z}=\frac{1-e^{-d z}}{1-e^{-z}} e^{-z} \\
& Q_{2}(z)=\sum_{i=d+1}^{k+1} e^{-i z}=\frac{e^{-d z}-e^{-(k+1) z}}{1-e^{-z}} e^{-z} \\
& Q_{3}(z)=\sum_{i=k+2}^{\infty} e^{-i z}=\frac{e^{-(k+1) z}}{1-e^{-z}} e^{-z} .
\end{aligned}
$$


By the theorem, given $P_{1}, P_{3}$, the maximum value $C\left(d, k ; P_{1}, P_{3}\right)$ of $\bar{H}$ under the given constraints is equal to $z_{0}\left(P_{1}, P_{3}\right) / \ln 2$, where $z_{0}=z_{0}\left(P_{1}, P_{3}\right)$ is the unique solution $z$ of

$-P_{1} \ln Q_{1}(z)-P_{2} \ln Q_{2}(z)-P_{3} \ln Q_{3}(z)$

$$
=-P_{1} \ln P_{1}-P_{2} \ln P_{2}-P_{3} \ln P_{3}
$$

with $P_{2}=1-P_{1}-P_{3}$.

We are particularly interested in the behavior of $C\left(d, k ; P_{1}, P_{3}\right)$ as a function of $P_{1}, P_{3}$ small. We first observe that for $P_{1}=P_{3}=0$, $P_{2}=1,(30)$ reduces to

$$
Q_{2}(z)=\frac{e^{-d z}-e^{-(k+1) z}}{1-e^{-z}} e^{-z}=1
$$

i.e., with $y=e^{z}$ to

$$
y^{k+2}-y^{k+1}-y^{k-d+1}+1=0 .
$$

This is the familiar equation associated with perfectly $(d, k)$-constrained sequences for which the capacity $C(d, k)$ is given by $\log _{2} y_{00}=z_{00} / \ln 2$, where $z_{00}$ is the unique positive solution of (31) and $y_{00}$ is $\exp \left(z_{00}\right)$. Since the $Q_{n}(z)$ are smooth functions of $z>0$, there holds for $z$ close to $z_{00}$

$$
Q_{n}(z)=Q_{n}\left(z_{00}\right)+\left(z-z_{00}\right) Q_{n}^{\prime}\left(z_{00}\right)+O\left(\left(z-z_{00}\right)^{2}\right) .
$$

From (30) it follows from some elementary considerations that

$$
z_{0}\left(P_{1}, P_{3}\right)=z_{00}+O\left(P_{1} \ln P_{1}+P_{3} \ln P_{3}\right) .
$$

For small $P_{1}, P_{3}$ we thus get that $z_{0}\left(P_{1}, P_{3}\right)$ satisfies

$$
\begin{aligned}
& \ln Q_{2}\left(z_{0}\left(P_{1}, P_{3}\right)\right) \\
& =P_{1} \ln P_{1}+P_{3} \ln P_{3}-\left(P_{1}+P_{3}\right)-P_{1} \ln Q_{1}\left(z_{00}\right) \\
& \quad-P_{3} \ln Q_{3}\left(z_{00}\right)+O\left(\left(P_{1}+P_{3}\right)\left(P_{1} \ln P_{1}+P_{3} \ln P_{3}\right)\right) .
\end{aligned}
$$

Hence, using that $Q_{2}\left(z_{00}\right)=1$

$$
Q_{2}\left(z_{0}\left(P_{1}, P_{3}\right)\right)=Q_{2}\left(z_{00}\right)+\Delta+\epsilon
$$

where

$$
\begin{aligned}
\Delta= & P_{1} \ln P_{1}+P_{3} \ln P_{3}-\left(P_{1}+P_{3}\right) \\
& -P_{1} \ln Q_{1}\left(z_{00}\right)-P_{3} \ln Q_{3}\left(z_{00}\right)
\end{aligned}
$$

and here and in the sequel $\epsilon$ denotes an $O$-term as in the third line of (34). Therefore,

$$
z_{0}\left(P_{1}, P_{3}\right)-z_{00}=\frac{\Delta}{Q_{2}^{\prime}\left(z_{00}\right)}+\epsilon
$$

and it follows that

$$
C\left(d, k ; P_{1}, P_{3}\right)-C(d, k)=\frac{z_{0}\left(P_{1}, P_{3}\right)-z_{00}}{\ln 2}=\frac{\bar{\Delta}}{Q_{2}^{\prime}\left(z_{00}\right)}+\epsilon
$$

with

$$
\begin{aligned}
\bar{\Delta}=\frac{\Delta}{\log _{2} 2}= & P_{1} \log _{2} P_{1}+P_{3} \log _{2} P_{3}-P_{1} \log _{2} Q_{1}\left(z_{00}\right) \\
& -P_{3} \log _{2} Q_{3}\left(z_{00}\right)-\frac{P_{1}+P_{3}}{\ln 2}
\end{aligned}
$$

Thus the difference $C\left(d, k ; P_{1}, P_{3}\right)-C(d, k)$ consists of a linear combination of terms $P_{1} \log _{2} P_{1}, P_{3} \log _{2} P_{3}, P_{1}, P_{3}$, and an $\epsilon$-error as $P_{1} \downarrow 0, P_{3} \downarrow 0$.

We next present two examples. The first example is merely meant to check that the theorem yields results that are in agreement with what one can also obtain by more elementary means. The second example is relevant for storage practice.

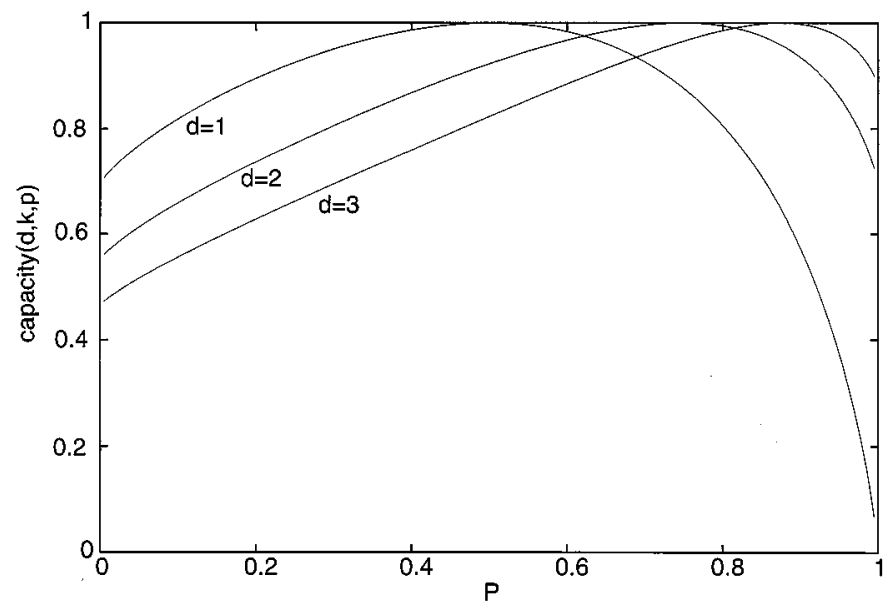

Fig. 1. The capacity $C\left(d, \infty ; P_{1}\right)$ of weakly $d$-constrained sequences as a function of the probability $P_{1}$ that the sequence violates the given $d$ constraint.

Example 1: Take $k=\infty$ so that the terms with index 3 disappear altogether. We have now

$$
Q_{1}(z)=\frac{e^{d z}-1}{e^{(d+1) z}-e^{d z}} \quad Q_{2}(z)=\frac{1}{e^{(d+1) z}-e^{d z}} .
$$

Equation (30) becomes

$$
\begin{aligned}
&-P_{1} \ln Q_{1}(z)-\left(1-P_{1}\right) \ln Q_{2}(z) \\
&=-P_{1} \ln P_{1}-\left(1-P_{1}\right) \ln \left(1-P_{1}\right)
\end{aligned}
$$

with solution $z_{0}\left(P_{1}\right)$ for $0 \leq P_{1} \leq 1$. Observe that

$$
\begin{aligned}
& C\left(d, \infty ; P_{1}=0\right)=\frac{z_{0}(0)}{\ln 2}=C(d, \infty) \\
& C\left(d, \infty ; P_{1}=1\right)=\frac{z_{0}(1)}{\ln 2}=C(0, d-1) .
\end{aligned}
$$

In Fig. 1 we have plotted $C\left(d, \infty ; P_{1}\right)$ as a function of $P_{1} \in(0,1)$ for $d=1,2,3$. It is seen that $C\left(d, \infty ; P_{1}\right)$ has maximum unity, and we shall show that this maximum occurs at $P_{1}=1-2^{-d}$. Indeed, using (40) and (41) we get for $z=z_{0}\left(P_{1}\right)$

$$
\begin{aligned}
\ln e^{d z}+\ln \left(e^{z}-1\right)- & P_{1} \ln \left(e^{d z}-1\right) \\
& =-P_{1} \ln P_{1}-\left(1-P_{1}\right) \ln \left(1-P_{1}\right) .
\end{aligned}
$$

Differentiating implicitly with respect to $P_{1}$ and setting

$$
\left(d z_{0}\left(P_{1}\right)\right) / d P_{1}=0
$$

we easily obtain

$$
P_{1}=1-e^{-d z_{0}\left(P_{1}\right)}
$$

Substituting this $P_{1}$ back into (43) we then exactly obtain $z_{0}\left(P_{1}\right)=$ $\ln 2$, as required. The above result can also be understood by noting that if the capacity is unity, the distribution is given by $p_{i}=2^{-i}, i \geq 1$ [3], so that the maximum occurs at $P_{1}=1-2^{-d}$.

As to (38) and (39), we let $y_{00}=\exp \left(z_{00}\right)$, so that $y_{00}$ is the unique solution $y>1$ of

$$
y^{d+1}-y^{d}=1
$$




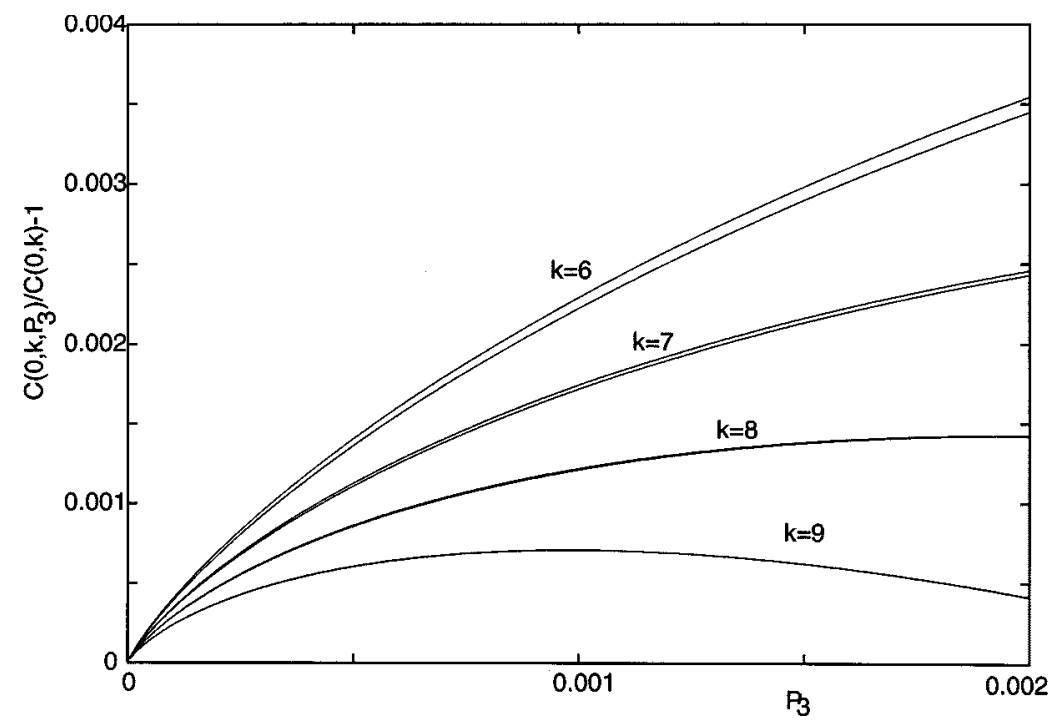

Fig. 2. The relative capacity gain $C\left(0, k ; P_{3}\right) / C(0, k)-1$ of weakly $k$-constrained sequences as a function of the probability $P_{3}$ that the sequence violates the given $k$-constraint. The upper curves are computed with full accuracy, while the lower curves are computed with approximation (55).

and we compute

$$
Q_{1}\left(z_{00}\right)=y_{00}^{d}-1 \quad Q_{2}^{\prime}\left(z_{00}\right)=-\left(d+1+y_{00}^{d}\right) .
$$

Therefore, there holds

$$
\begin{aligned}
C\left(d, \infty ; P_{1}\right)-C(d, \infty) \\
=-\frac{P_{1}\left(\log _{2} P_{1}-\log _{2}\left(y_{00}^{d}-1\right)-1 / \ln 2\right)}{d+1+y_{00}^{d}}
\end{aligned}
$$

and, indeed, Fig. 1 shows a $-P_{1} \log _{2} P_{1}$-behavior of $C\left(d, \infty ; P_{1}\right)-$ $C(d, \infty)$ near $P_{1}=0$.

Example 2: We now consider the case that $d=0$ and $k \geq 6$, so that

$$
T=\{1, \cdots, k+1\} \cup\{k+2, k+3, \cdots\}=S_{2} \cup S_{3}
$$

where the sets $S_{2}$ and $S_{3}$ are assigned probabilities $P_{2}$ and $P_{3}, P_{2}+$ $P_{3}=1$, with $P_{3}$ small. In fact, this is Example 1 with $d$ replaced by $k+1, S_{1}$ replaced by $S_{2}, S_{2}$ replaced by $S_{3}$, and $P_{1}$ replaced by $P_{2}=1-P_{3}$. Hence we consider Fig. 1 at the far right-hand side of the $P_{1}$-axis. Accordingly, we have

$$
Q_{2}(w)=\frac{e^{(k+1) w}-1}{e^{(k+2) w}-e^{(k+1) w}} \quad Q_{3}(w)=\frac{1}{e^{(k+2) w}-e^{(k+1) w}}
$$

where we have written $w$ rather than $z$ to avoid confusion with the $z$ in Example 1. Equation (30) becomes

$$
\begin{aligned}
-\left(1-P_{3}\right) \ln Q_{2}(w)- & P_{3} \ln Q_{3}(w) \\
& =-\left(1-P_{3}\right) \ln \left(1-P_{3}\right)-P_{3} \ln P_{3}
\end{aligned}
$$

the solution $w$ of which we denote by $w_{0}\left(P_{3}\right)$. For the corresponding capacity at $P_{3}=0$ we have

$$
C\left(0, k ; P_{3}=0\right)=\frac{w_{0}(0)}{\ln 2}=C(0, k) .
$$

Denoting $w_{0}(0)=w_{00}$ and $x_{00}=\exp \left(w_{00}\right)$ we have that $x_{00}$ is the unique solution $x>1$ of the equation

$$
x^{k+2}-2 x^{k+1}+1=0, \quad \text { i.e., } x=2-\frac{1}{x^{k+1}} .
$$

The formulas (38) and (39) yield for $P_{3} \rightarrow 0$

$$
\begin{aligned}
& C\left(0, k ; P_{3}\right)-C(0, k) \\
& \quad=\frac{P_{3} \log _{2} P_{3}-P_{3} \log _{2} Q_{3}\left(w_{00}\right)-P_{3} / \ln 2}{Q_{2}^{\prime}\left(w_{00}\right)}+\epsilon .
\end{aligned}
$$

We compute, using (52) repeatedly

$$
\begin{aligned}
& Q_{3}\left(w_{00}\right)=\frac{1}{x_{00}^{k+1}-1} \\
& Q_{2}^{\prime}\left(w_{00}\right)=-2+\frac{k}{x_{00}^{k+1}-1} .
\end{aligned}
$$

Finally, when $k \geq 6$ we have (see the second formula in (52)) that $x_{00}$ is close to 2 , where $Q_{3}\left(w_{00}\right) \approx x_{00}^{-k-1}, Q_{2}^{\prime}\left(w_{00}\right) \approx-2$. This yields the approximation as $P_{3} \rightarrow 0$

$$
\begin{aligned}
& C\left(0, k ; P_{3}\right)-C(0, k) \\
& \quad \approx \frac{1}{2}\left(-P_{3} \log _{2} P_{3}-(k+1) P_{3} \log _{2} x_{00}+P_{3} / \ln 2\right) .
\end{aligned}
$$

In Fig. 2 we have plotted the relative capacity gain

$$
\frac{C\left(0, k ; P_{3}\right)-C(0, k)}{C(0, k)}
$$

for $k=6,7,8,9$ and $P_{3} \in(0,0.002]$. It is seen that (56) exhibits the expected $\frac{1}{2} P_{3} \log _{2} P_{3}$ behavior for $P_{3}$ very near to zero, but that the linear terms at the right-hand side of (55) dominate the $P_{3} \log _{2} P_{3}$ term from $P_{3}=2^{-k-1}$ onwards (see end of Example 1). As we can see in Fig. 2, the approximation given in (55) is quite accurate, especially for larger values of $k$.

We finally consider the case that, with $d$ and $k$ as before, the set $T=\{1,2, \cdots\}$ is partitioned as

$$
T=\{1, \cdots, d, k+2, k+3, \cdots\} \cup\{d+1, \cdots, k+1\}=S_{1} \cup S_{2}
$$

so that sets $S_{1}, S_{3}$ in (25) are merged into one set $S_{1}$, with probabilities $P_{1}, P_{2}$ assigned to $S_{1}, S_{2}$. The analysis for this case proceeds along 
the same lines as for the partitioning of $T$ as in (25). In particular, we have now

$$
\begin{aligned}
& Q_{1}(z)=\frac{1-e^{-d z}+e^{-(k+1) z}}{1-e^{-z}} e^{-z} \\
& Q_{2}(z)=\frac{e^{-d z}-e^{-(k+1) z}}{1-e^{-z}} e^{-z}
\end{aligned}
$$

(the same $Q_{2}$ as in (28)), and $C\left(d, k ; P_{1}=0\right)=C(d, k)$. Also, $z_{00}$ is the same as before, and for the behavior of $C\left(d, k ; P_{1}\right)$ as $P_{1} \downarrow 0$ we now find

$$
\begin{aligned}
C\left(d, k ; P_{1}\right)-C(d, k)= & \frac{P_{1}\left(\log _{2} P_{1}-\log _{2} Q_{1}\left(z_{00}\right)-1 / \ln 2\right)}{Q_{2}^{\prime}\left(z_{00}\right)} \\
& +O\left(P_{1}^{2} \log _{2} P_{1}\right)
\end{aligned}
$$

\section{CONCLUSIONS}

We have presented an analytic expression for the maximum of the normalized entropy $-\sum_{i \in T} p_{i} \ln p_{i} / \sum_{i \in T} i p_{i}$ under the condition that $T$ is the disjoint union of sets $S_{n}$ of positive integers that are assigned probabilities $P_{n}, \sum_{n} P_{n}=1$. This result has been applied to compute the capacity of weakly $(d, k)$-constrained sequences that are allowed to violate the $(d, k)$-constraint with a given (small) probability.

\section{REFERENCES}

[1] K. A. S. Immink, "Weakly constrained codes," Electron. Lett., vol. 33, no. 23, pp. 1943-1944, Nov. 1997.

[2] —- Codes for Mass Data Storage Systems. Amsterdam, The Netherlands: Shannon Foundation Publishers, 1999.

[3] C. E. Shannon, "A mathematical theory of communication," Bell Syst. Tech. J., vol. 27, pp. 379-423, July 1948

\section{Time-Varying Encoders for Constrained Systems: An Approach to Limiting Error Propagation}

Jonathan J. Ashley, Member, IEEE, and Brian H. Marcus, Fellow, IEEE

\begin{abstract}
Time-varying encoders for constrained systems are introduced. The approach generalizes the state-splitting (ACH) algorithm in a way that yields encoders consisting of multiple phases, with encoding proceeding cyclically from one phase to the next. The framework is useful for design of high-rate codes with reduced decoder error propagation and reduced complexity.
\end{abstract}

Index Terms-Constrained systems, finite-state encoder, input-restricted channel, PRML, sliding-block decoder, state splitting.

\section{INTRODUCTION}

Constrained coding is a special kind of channel coding in which unconstrained user sequences are encoded into sequences that are required to satisfy certain hard constraints such as runlength limits [10], [14], [13].

Manuscript received December 29, 1998.

J. J. Ashley is with Infineon Technologies, Santa Cruz, CA 95060 USA (e-mail: Jonathan.Ashley@infineon.com).

B. H. Marcus is with IBM Almaden Research Center, San Jose, CA 95120 USA (e-mail: marcus@almaden.ibm.com).

communicated by R. M. Roth, Associate Editor for Coding theory.

Publisher Item Identifier S 0018-9448(00)03101-1.
In a finite-state encoder, arbitrary user data sequences are encoded to constrained data sequences via a finite-state machine. The encoder is said to have rate $p: q$ if at each step of the encoding process, one $p$-tuple of user data is encoded to one $q$-tuple of constrained data in such a way that the concatenation of the encoded $q$-tuples obeys the given constraint. For the purposes of limiting decoder error propagation, decoding is usually implemented via a sliding-block decoder. One method of constructing finite-state encoders is the state-splitting algorithm (also called the ACH algorithm) [1], [14]. The main purpose of our correspondence is to show how to adapt the state-splitting algorithm to the time-varying setting and to use it as an outline for constructing high-rate codes with limited error propagation.

The state-splitting algorithm begins with a representation of the desired constraint and iteratively constructs a sequence of graphs, ultimately arriving at a graph that can be used as an encoder. The last step in the construction is the data-to-codeword assignment, where input labels (or tags) are assigned to the edges of this graph. At each state this amounts to a 1-1 assignment between the set of all binary $p$-tuples and $2^{p}$ of the outgoing edges. The choice of assignment can significantly affect the complexity and performance of the code.

When $p$ is relatively small, one can usually find a reasonably good assignment simply by ad hoc experimentation. But when $p$ is relatively large, there are far too many possible assignments and a poor choice could lead to a very costly implemenatation. Indeed, for large $p$, the data-to-codeword assignment becomes more of the heart of the code construction problem. Recently, the data recording industry has been moving toward detection schemes that can function well at very high code rates such as $16: 17,24: 25$, and $32: 33$. Most such codes that can be found in the literature have been designed by clever ad hoc procedures.

The design of such a code may appear to be somewhat mind-boggling at first. After all, a rate $24: 25$ code would involve some assignment of all $2^{24}$ binary strings of length 24 . In much of the previous work, this difficulty is overcome by "breaking down" the coding problem into smaller subproblems: say, by partitioning the coordinates into a few smaller groups, designing an encoding strategy on each of these groups and then putting together the resulting encoded strings in a way that satisfies the constraint; see, for example, [17], [12], [5], [4], [15]. One can think of the constraint and the encoders as changing from each one of these groups to another. In connection with this, we mention that periodically time-varying constraints have been introduced in [3] and [6].

Inspired by this work as well as our own independent efforts to design low-complexity codes, we show in this correspondence how to adapt the state-splitting algorithm to the setting of periodically timevarying encoders. For instance, we could construct a rate $16: 19$ code by employing two different finite-state encoders in alternating phases: a rate $8: 9$ phase and a rate $8: 10$ phase; this can potentially yield a low-complexity encoder. But there is another advantage. For a generic block-decodable 16:19 code, an isolated channel error may corrupt one 16-bit block, equivalently two user bytes. If such a code were constructed as a two-phase rate $8: 9 / 8: 10$ block-decodable code (i.e., each 9-bit block and 10-bit block decodes directly to a user byte), then such an error could corrupt only one user byte. If the latter were not possible, one might try to construct a two-phase $8: 9 / 8: 10$ sliding-block-decodable code with sliding window consisting of two blocks (a 9-bit block followed by a 10-bit block and vice versa). In this case, an isolated channel error could corrupt two user bytes. However, a 2-bit channel error (such as a bit shift) could corrupt only three user bytes, whereas in a generic block-decodable $16: 19$ code it might corrupt four user bytes. 